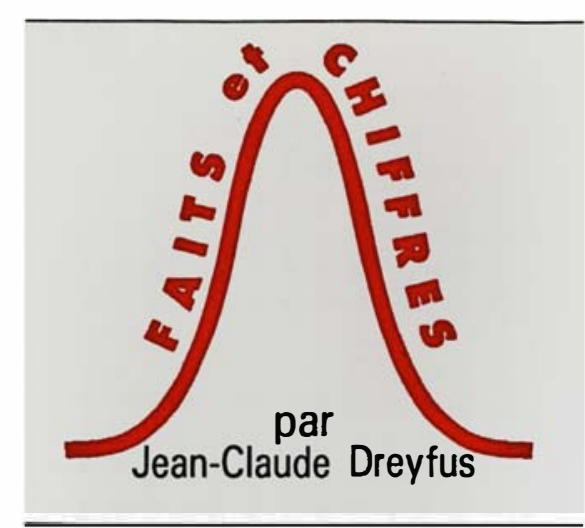

Source : Calot G. La fécondité en Europe : analogies et différences. Population et Sociétés. INED (Institut national d'études démographiques). Janvier 1992, n 264.

\title{
La fécondité en Europe
}

$\mathbf{U}$ ne controverse assez rude a récemment secoué les milieux de la démographie, science en principe statistique et neutre, mais dont les conclusions peuvent être importantes lorsque sont face à face des pays à faible fécondité, comme ceux de l'Europe, et d'autres dont la natalité est galopante. On peut en effet adopter une approche transversale, comptant le nombre des naissances annuelles et en déduire chaque année l'indice conjoncturel de fécondité ; ou une approche longitudinale, considérant les générations successives, et synthétisant le comportement de chacune au moyen de la descendance finale. Ces deux approches sont complémentaires, l'une donnant des renseignements immédiatement utilisables, l'autre à retardement (on s'arrête actuellement au comportement des femmes nées entre 1950 et 1960), mais permettant peut-être des prévisions plus solides.

La figure 1 présente l'évolution de l'indicateur conjoncturel de fécondité de 1950 à 1990 en Europe. On voit - c'était connu - un maximum vers 1965, suivi d'une chute. Mais on peut distinguer trois tendances récentes selon les pays : ceux de l'Europe de l'Ouest, y compris l'Angleterre, sont pratiquement stables depuis 1980 à des niveaux divers mais tous peu élevés (la RFA, record de dénatalité pendant dix ans, remonte légèrement). En Europe du Sud, la baisse, plus tardive à se manifester, se poursuit encore et atteint des niveaux inconnus auparavant. Le fait le plus nouveau concerne les pays scandinaves, qui tous, à partir de 1980, voient leur niveau remonter, notamment la Suède, qui a atteint et franchi la barre de deux enfants par femme.
Peut-on interpréter ces variations au vu de la courbe de la descendance finale des générations, présentée à partir de 1920 dans la figure 2 ? Cette courbe s'est stabilisée pour la Suède dès 1940 au voisinage de deux enfants par femme, dix ans plus tard pour la France $(2,1)$ et la Finlande $(1,8)$. La Belgique en est proche $(1,8)$. On peut la prévoir pour 1960 environ pour l'Angleterre (1,9 probable), alors qu'à cette date la RFA n'est guère au-dessus de 1,5.

On sait que cette stabilisation après baisse résulte de deux phénomènes : la baisse réelle de la fécondité, et le décalage qui se produit depuis plusieurs décennies puisque les femmes ont tendance à retarder leurs grossesses. Il se crée ainsi, pendant la période de décalage, une accentuation de la baisse qui cesse quand cette période se termine. Le résultat à tout moment vient de la conjonction des deux facteurs, comme on le voit en comparant les deux pays les mieux étudiés, Suède et France : dans les deux pays, la hausse des taux aux âges élevés de fécondité continue, mais, tandis que la baisse chez les femmes jeunes se poursuit en France, elle a cessé en Suède d'où l'indice conjoncturel plus élevé de ce dernier pays.

On voit ainsi que, si la démographie permet d'interpréter les résultats, elle ne saurait en revanche prétendre extrapoler, même à moyen terme : il est impossible, par exemple, de savoir si la fécondité des femmes les plus jeunes cessera de décroître en France, permettant une remontée globale. Et qui eût dit, il y a quinze ans, que les pays catholiques d'Europe du Sud tomberaient à 1,3-1,4 enfant par femme, une chute dont rien n'annonce la fin? 


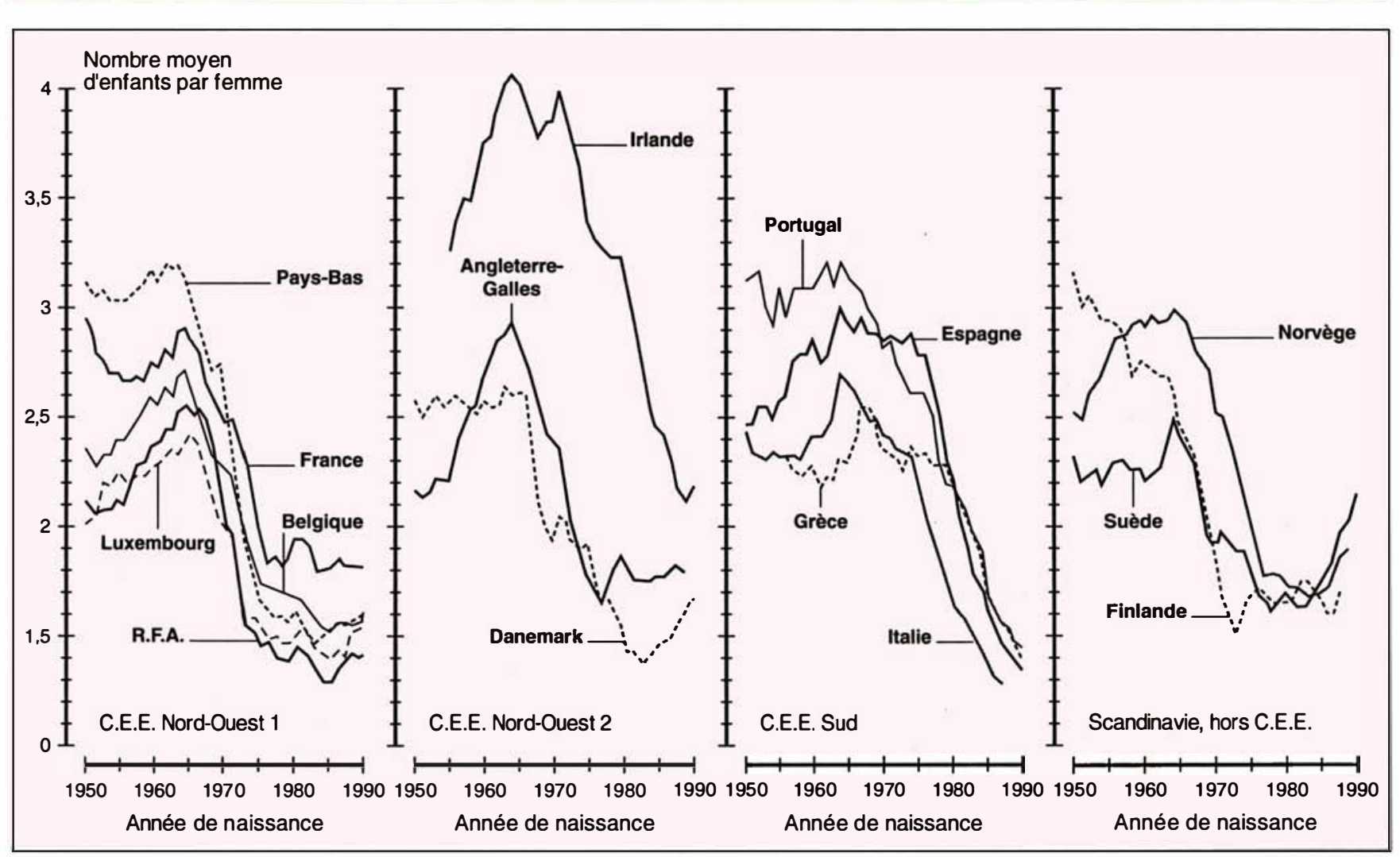

Figure 1. Évolution, depuis 1950, de l'indicateur conjoncturel de fécondité.

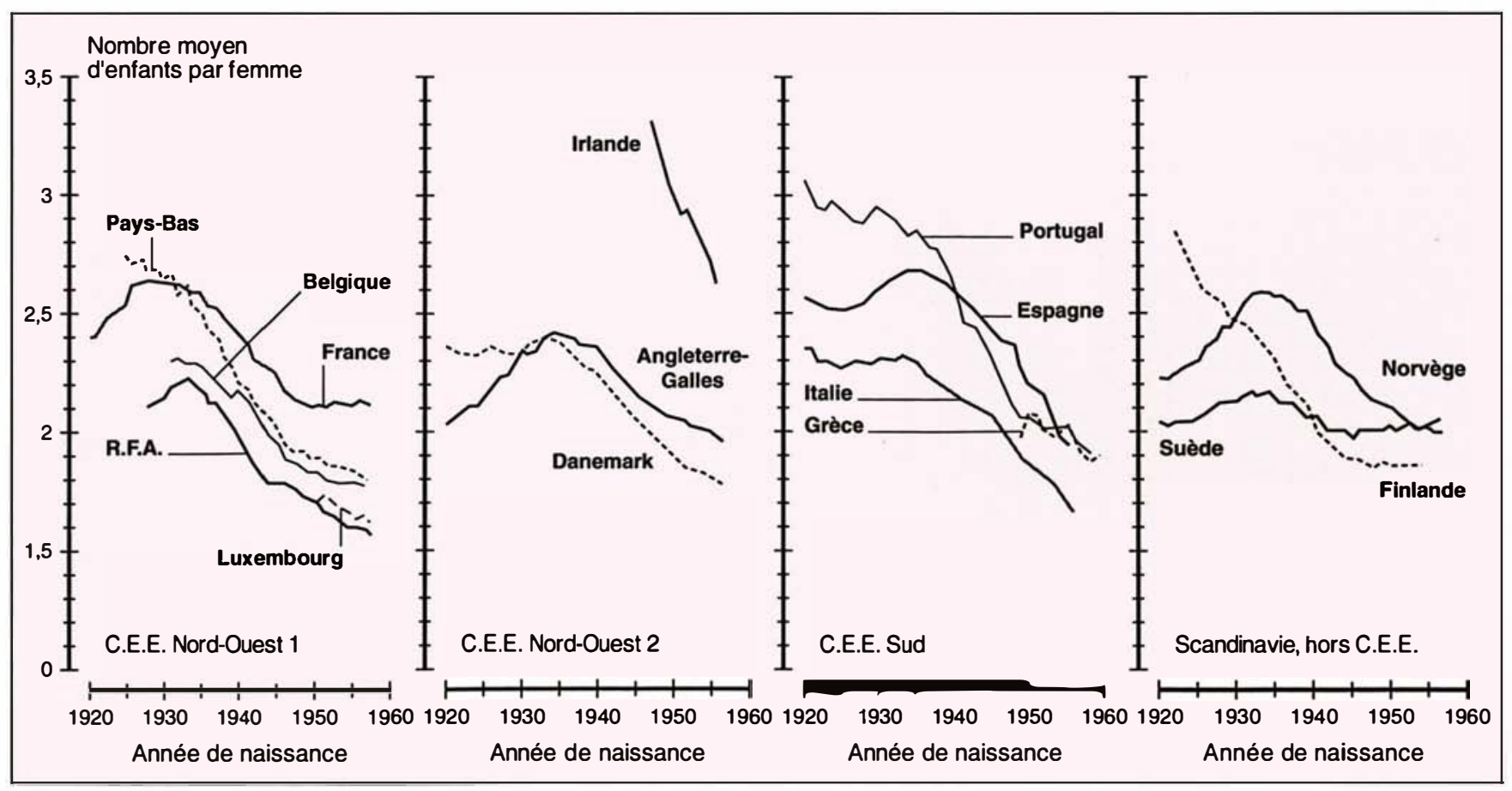

Figure 2. Évolution, depuis 1920, de la descendance finale des générations. 